\title{
Fabrication and Properties of Dense Silicon Carbide Ceramic via Gel-Casting and Gas Silicon Infiltration
}

Lu-ming Huang, Rong-jun Liu, Yan-fei Wang, Chang-rui Zhang, Xian-hai Long, Ying-bin Cao

(Science and Technology on Advanced Ceramic Fibers and Composites Laboratory, National University of Defense Technology, Changsha 410073, P.R China)

\begin{abstract}
The dense Silicon Carbide ( $\mathrm{SiC}$ ) ceramics are fabricated by means of gel-casting and gas silicon infiltration (GSI) using carbon black and $\alpha$-SiC as raw materials. We have successfully introduced a new initiator AIBA which is very suitable to aqueous gel-casting system containing carbon black, overcoming the problems posed by the conventionally used initiator. We have investigated the influences of the monomer acrylamide (AM) content, the ratio of the monomer to crosslinking agent AM/MBAM content, the particle size distribution and the solid content on the mechanical and structural properties of samples. The result show that, the linear shrinkage of the green body can be reduced to $1.0 \%$ and its bending strength can reach $59.2 \mathrm{MPa}$ at the optimized gel-casting process that has an AM content of $25 \mathrm{wt} \%$, an AM to MBAM ratio of 12, a SiC particle distribution of $3 / 2$ and a solid content of 60 vol. $\%$. After the GSI process, the bending strength and elastic modulus of the final products from such green bodies can reach $245 \mathrm{MPa}$ and $220 \mathrm{GPa}$ respectively. The study highlights that the combined application of the gel-casting and the GSI processes can produce high-quality silicon carbide ceramics that are suitable in the space optical applications.
\end{abstract}

Key words: A. Powders: gas phase reaction; C. Mechanical properties; D. SiC; Gel-casting 


\section{Introduction}

Optical system plays a crucial role in the space collecting information. As a key component in such optical systems, the space mirror has drawn intensive attentions of researchers. The candidate materials for space mirrors need to possess a high flexural strength, a high elastic modulus (>200 GPa), as well as a dense structure without any holes, etc. Currently, the silicon carbide ( $\mathrm{SiC}$ ) is considered as an ideal material for space mirrors due to a combination of its desirable properties for space systems, such as high mechanical properties, good optical performance, corrosion resistance, etc [1-4]. There are a wide variety of methods to make SiC materials among which the gas silicon infiltration (GSI) is the most promising process due to its short preparation period, low sintering temperature, minimum sintering shrinkage and low cost. In a typical GSI process, the gaseous silicon reacts with carbon to form $\beta$-SiC at a temperature range of $1500-1700{ }^{\circ} \mathrm{C}$ with the existence of carbon black [5-6].

Prior to the GSI process, a carbon green body with an appropriate porosity need to be prepared. In the current study, we select the gel-casting method to make such green carbon bodies due to its advantages such as to make green bodies with near-net-shape, high strength, high quality, and high density, etc. Currently, a commonly used aqueous gel-casting system includes acrylamide (AM) as the monomer, N,N'-Methylenebisacrylamide (MBAM) as the crosslinking agent and ammonium persulphate (APS) as the initiator [7-14]. Unfortunately, the addition of carbon black to this system exerts a detrimental effect on the gelation. The carbon black is well known of composing of layered hexagonal graphite microcrystalline, 
which has a strong capturing effect on the radical $\cdot \mathrm{SO}_{4}{ }^{-}$released by the initiator APS. In addition, this effect can in turn promote the release of the radicals according to Le Chatelier's principle. Hence the gelation reaction cannot be controlled, i.e. the gelation time tends to be less than 5minutes. Moreover, the gelation acceleration effect becomes more apparent with higher carbon contents. Clearly, such uncontrollable gelation processes cannot satisfy the requirement of the casting process, which should have a gelation time ideally more than 30minutes. In order to address this problem, Chen et al added acetylacetone (Acac) into the slurry so as to slow down the gelation reaction, however, Acac did not work when the carbon content was over 15 wt.\%. In another attempt, Zhang et al used dextrin instead of carbon black, but the sintering temperature became too high, i.e. around $2200{ }^{\circ} \mathrm{C}$ [15-18], which becomes inappropriate.

In this study, the direct initiative is to address the initiator issue. We select a new initiator 2,2'-Azobis (2-methylpropionamidine) dihydrochloride (AIBA) to replace APS and investigate the influence of factors such as the AM/MBAM content, particle size distribution and solid content on the gel-casting process. The following findings are unveiled. Firstly, the carbon black has much weaker capability to capture radicals released by AIBA. Secondly, the concentration of initiators AIBA and the reaction temperature control the decomposition of AIBA. Hence, the gelation reaction can be controlled at ease, suggesting AIBA is appropriate as an initiator gel-casting system that contains carbon. 


\section{Experimental}

\subsection{Gel-casting process}

The green body was fabricated by gel-casting. Firstly, AM and MBAM (Chinese Medicine Chemical Reagent Co., Ltd.) were dissolved in deionized water to form a solution. Secondly, the carbon black (N550, $35 \mathrm{~m}^{2} / \mathrm{g}$, China United Rubber (Group) Company) and SiC powders (Qingzhou Shandong fine powder Co., Ltd) were added to the above solution to form slurry, with the addition of PVP (polyvinyl pyrrolidone, Chinese Medicine Chemical Reagent Co., Ltd.) and TMAH (Tetramethylammonium hydroxide, Chinese Medicine Chemical Reagent Co., Ltd.) as the dispersants of C and $\mathrm{SiC}$ respectively. Subsequently, the above slurry is subjected to $8 \mathrm{~h}$ ball-mixing and 30 min vacuum-degassing, AIBA (Shanghai Aladdin Bio-chem Technology Co., Ltd.) was added to the slurry. Afterwards, the slurry was poured to the mould that is $60 \mathrm{~mm}$ in diameter and $10 \mathrm{~mm}$ in height and the gelation reaction took place. Finally, the green body was dried to constant weight at room temperature.

\subsection{Gas silicon infiltration process}

Dense $\mathrm{Si} / \mathrm{SiC}$ ceramics were prepared by the GSI method. The above-obtained green body was buried in the silicon powders in a furnace and then heated up to $1500{ }^{\circ} \mathrm{C}$ in $5 \mathrm{~h}$ in the argon atmosphere to turn the solid $\mathrm{Si}$ to liquid phase. Subsequently the furnace was fired to $1700{ }^{\circ} \mathrm{C}$ in $1 \mathrm{~h}$ in vacuum and dwelled at this temperature for $2 \mathrm{~h}$, during which the liquid Si turned to gaseous phase and the silicon and carbon reaction took place. 


\subsection{Samples characterization}

Differential scanning calorimetry (DSC/DTA-TG, Netzsch STA 449 F3 Jupiter) was used to analyze the gelation reaction and to calculate the reaction heat. $\mathrm{The} \mathrm{Al}_{2} \mathrm{O}_{3}$ serves as the reference material, with the heating rate as $10{ }^{\circ} \mathrm{C} / \mathrm{min}$ and the dwelling time as $60 \mathrm{~min}$.

The mechanical properties are evaluated by the three-point bending test. The samples were cut and polished to $40 \mathrm{~mm}$ in length, $4 \mathrm{~mm}$ in width and $3 \mathrm{~mm}$ in thickness. The length and width directions were parallel to warp and weft directions, respectively. During the three-point bending test, the ratio of span to height was kept as 15 and the crosshead speed was $0.5 \mathrm{~mm} / \mathrm{min}$. The flexural strength $\sigma_{\mathrm{b}}$ and elastic modulus $\mathrm{E}_{\mathrm{b}}$ are calculated according to Eq.1 and Eq.2 respectively.

$$
\begin{aligned}
& \sigma_{\mathrm{b}}=\frac{3 P \mathrm{~L}}{2 b h^{2}} \\
& E_{\mathrm{b}}=\frac{\mathrm{L}^{3}}{4 b h^{3}} \cdot \frac{\Delta P}{\Delta f}
\end{aligned}
$$

Where $\mathrm{P}$ is the maximum load, $\mathrm{L}$ is the span, $\mathrm{b}$ is the specimen width, $\mathrm{h}$ is the specimen thickness, and $\Delta \mathrm{P} / \Delta \mathrm{f}$ is the slope of the linear portion of the load-displacement curve.

The microstructures of the specimens before and after sintering were examined by the scanning electron microscopy (SEM, FEI Quanta-200).

The phase and composition of the samples were identified by the X-ray diffraction (XRD, D8 Advance), with the Cu-ka radiation. The samples were scanned from 10 to $80^{\circ}$, with $0.01^{\circ}$ as the step length and holding $1 \mathrm{~s}$ at each step. The 
chemical compositions of the samples were analyzed by the X-ray photoelectron spectroscopy (XPS, Escalab 250Xi), using $12 \mathrm{kV}$ Al-Ka as the exciting light source, with a power of $72 \mathrm{~W}$ and a resolution of $0.5 \mathrm{eV}$. Prior to the test, samples were subjected to the sputtering $\mathrm{Ar}^{+}$for $10 \mathrm{~min}$ so as to reduce the surface contamination.

\section{Results and discussion}

\subsection{Preparation of high solid content slurry}

Fig.1(a) shows the effect of the monomer AM content in water on the viscosity of the slurry. As shown, the viscosity of the slurry increases dramatically with the increase of the AM content, which can be attributed to the following reasons. Firstly, with the addition of the monomer AM, the volume of the solution expands. For instance, the addition of each gram monomers gives $0.92 \mathrm{ml}$ solution volume expansion. By contrast, as we keep the solid content constant, the content of deionized water in the slurry decreases with the increase of the AM content. Secondly, with the addition of the monomer AM, the organic molecules interaction become more violent, which can undoubtedly lead to the increase of the viscosity.

Fig.1(b) shows the effect of particle size distribution on the viscosity of slurry. The viscosity of the slurry initially decreases and then increases, with the F240/F1200 ratio at $3 / 2$ reaching the lowest value. According to Andreason classical close packing theory, the content of particles with different sizes can be calculated by Eq.3,

$$
\mathrm{U}(\mathrm{D})=\left(\frac{\mathrm{D}}{D_{\max }}\right)^{m}
$$

where $D_{\max }$ is the diameter of the maximum particle, $U(D)$ is the mass fraction of the particles that have a diameter of $\mathrm{D}$, and $\mathrm{m}$ refers to the model parameter. Based on 
the simulation results, when $\mathrm{m}=0.37$, the void fraction of the model reaches the minimum. In this study, in order to prepare high solid content slurry, we employed two kinds of SiC powders with different particle sizes: the coarse ones (refereed as F240) with an average diameter of $43 \mu \mathrm{m}$ and the fine ones (referred as F1200) with an average diameter of $3.8 \mu \mathrm{m}$. Accordingly, Eq.3 gives a U(D) value of 0.41 with $D_{\max }=43, D=3.8, m=0.37$. That is to say, when $\mathrm{F} 240 / \mathrm{F} 1200=3 / 2, \mathrm{SiC}$ powders come to the most compact stack state, and it can be considered that coarse powders are closely packed, fine particles are filled in the space homogeneously. In this case, there is no particle aggregation of only F240 or F1200, and it is generally known that particle aggregation can cause the increase of viscosity. As is shown in Fig.1(c), when F240/F1200=3/2, the viscosity of slurry come to a minimum.

Fig.1(c) demonstrates the effect of solid content on the viscosity of the slurry. As seen, the viscosity increases steadily with the increase of the solid content. It is well known that the slurry becomes too viscous to be used in the gel-casting process when the viscosity of the slurry exceeds $1.5 \mathrm{~Pa} \cdot \mathrm{s}$. On the other hand, pores will appear in the green body when the solid content is less than 55 vol.\%. Considering the above two factors, the solid content of 60 vol. $\%$ is used in the current study.

\subsection{Process optimization of the gelation reaction}

Fig.2(a) shows the effect of the content of initiator AIBA on the polymerization reactions. As shown, a significant change of the DSC value $(\sim 0.8 \mathrm{~W} / \mathrm{g})$ after $50 \mathrm{mins}$ can be detected for the slurry that has an AIBA content of $20 \mu \mathrm{l}$ per gram of slurry. In contrast, a small change or even no change of the DSC value can be found for the 
slurry that has an AIBA content of 15 or $10 \mu$ per gram of slurry, suggesting an incomplete or even no polymerization reaction takes place in them. In addition, the conversion rate of the monomer AM can be calculated by the following equation.

$$
\alpha=\frac{m_{t}}{m_{0}}=\frac{Q_{t}}{Q_{0}}=\frac{Q_{t}}{\Delta H_{0}}=\frac{1}{1000} \cdot \frac{\frac{M}{c} \cdot A}{\Delta H_{0}}
$$

In which $\alpha$ is conversion rate, $\mathrm{m}$ is the mass of the monomer involved in the reaction, $\mathrm{Q}_{\mathrm{t}}(\mathrm{kJ} / \mathrm{mol})$ and $\mathrm{Q}_{0}\left(\Delta \mathrm{H}_{0}=82.8 \mathrm{~kJ} / \mathrm{mol}\right)$ refer to the actual heat and the theoretical value respectively, $\mathrm{M}(71.08 \mathrm{~g} / \mathrm{mol})$ is the relative molecular mass of AM, A $(\mathrm{J} / \mathrm{g})$ is the area of the DSC curve, and c (51.03 in this study) is the mass percentage of AM in slurry.

According to Eq.4, the conversion rate can be calculated as $98.77 \%, 34.46 \%$ and $3.94 \%$ respectively for the slurry that has an AIBA content of 20,15 or $10 \mu$ per gram of slurry. This can be accounted for as follows: When the initiator AIBA content is insufficient for the case of $15 \mu \mathrm{l}$ per gram of slurry, the concentration of radicals generated is inadequate for the complete polymerization reaction and thus some monomers do not take part in the reaction. As for the case of $10 \mu 1$ per gram of slurry, the radicals in the slurry created cannot even initiate the polymerization reaction. In addition, the gelation time can be also obtained from Fig.2(a). As shown, After 50 min, the DSC value almost reaches zero, suggesting that 50 min can be taken as termination of the gelation reaction.

Fig.2(b) exhibits the effect of the reaction temperature on the polymerization reaction. The reaction rate constant can be calculated according to the Arrhenius equation: 


$$
\mathrm{k}=\mathrm{A} \cdot \exp \left(-\frac{E}{R T}\right)
$$

In which $\mathrm{E}$ is activation energy (for AIBA, $\mathrm{E}$ is $124 \mathrm{~kJ} / \mathrm{mol}$ ), $\mathrm{R}$ is the molar gas constant that is $8.314 \mathrm{~J} \cdot \mathrm{g}^{-1} \cdot \mathrm{mol}^{-1}$, and $\mathrm{T}$ is absolute temperature. On the basis of the calculation of Eq.5, at $70{ }^{\circ} \mathrm{C}$ the $\mathrm{k}$ value is 3.69 times of that at $60^{\circ} \mathrm{C}$ and 14.77 times of that at $50{ }^{\circ} \mathrm{C}$, indicating that the reaction rate constant $\mathrm{k}$ has an exponential relationship with temperature. Therefore, in this study we select $70{ }^{\circ} \mathrm{C}$ as the reaction temperature.

\subsection{Drying behavior of green body}

The drying behavior plays a central role in the gel-casting process. Two factors are widely used to evaluate the drying behavior: the linear shrinkage and the flexure strength of the green body. Fig. 3 and Fig. 4 show the variations of the linear shrinkage and the flexure strength of the green body with the variables of the monomer AM content, the content ratio of AM to MBAM, the ratio of particle distributions F240 to F1200, and the solid content respectively. In the following contexts, in order to obtain an optimal condition of gel casting process, we are to discuss the influence of each of the above variable on the linear shrinkage and flexure strength of the green body.

From Fig.3(a), with the increase of the monomer AM content, the linear shrinkage of the green body initially decreases noticeably to the point of $25 \mathrm{wt} . \%$ of the AM content, beyond which the linear shrinkage begins to increase gently. On the other hand, from Fig.4(a), the bending strength of the green body increases monotonously with the increase of the AM content, although the increasing extent becomes smaller beyond $25 \mathrm{wt} \%$ of the AM content. Therefore, from the above two 
perspectives, $25 \mathrm{wt} . \%$ of the AM content seems to be the optimal monomer content. The reasons can be accounted for as follows. Firstly, when the monomer AM content is low, the polyacrylamide cannot yet form a complete network and thus, such an incomplete network is not strong enough to resist the capillary force exerted by the loss of the water in the drying process. Hence, the shrinkage tends to be relatively large whilst the bending strength tends to be low. Secondly, with an increase of the $\mathrm{AM}$ content, although the quantity is not enough to form a complete network when the AM content is less than $25 \mathrm{wt} \%$, the linear shrinkage decreases steadily whilst the flexure strength increases continuously, attributing to that the polyacrylamide itself possesses some strength. Thirdly, when the AM content reaches the critical point, i.e. around 25 wt.\%, the complete network is thought to have been formed. Upon the addition of the extra monomers, some useless networks might be formed, which limits the load of ceramic powders in the original network. Driven by the capillary force, these unloaded powders are rearranged, which severely damages the loading capability of such networks. The above explanations are further supported by the microstructure details of the green bodies, as shown in Fig.5. As seen, the green bodies with 15 wt.\% of the AM content have the most cracks and holes, whilst those with 20 wt. $\%$ have less defects. By contrast, those with 25 wt. $\%$ and 30 wt.\% of the AM content are almost dense, free of cracks and holes.

From Fig.3(b), with the increase of the AM/MBAM ratio, the shrinkage of the green body initially decreases and then increases, with the AM/MBAM ratio at 12 reaching the lowest value. On the other hand, From Fig.4 (b), with the increase of the 
AM/MBAM ratio, the flexure strength initially increases and then decreases, with the AM/MBAM ratio at 12 reaching the lowest value. This indicates the optimal AM/MBAM ratio is 12 . When the content of crosslinking agent MBAM is low, i.e. high AM/MBAM ratio, the polyacrylamide tends to be weakly crosslinked, which results in the formation of an incomplete three-dimensional structure that usually has a low bending strength and high linear shrinkage. On the contrary, when the content of the crosslinking agent MBAM is too high, i.e. low AM/MBAM ratio, the crosslinking degree in some parts of the polyacrylamide becomes so high that the network tends to fall apart and become brittle.

From Fig.3(c), with the increase of the F240/F1200 ratio, the linear shrinkage of the green body firstly decreases and then increases, with the F240/F1200 ratio at 3/2 reaching the lowest value. On the other hand, from Fig.4(c), with the increase of the F240/F1200 ratio, the bending strength of the green body firstly increases and then slightly decreases, with the F240/F1200 ratio at 3/2 reaching the highest value. This undoubtedly suggests the particle distribution of $3 / 2$ is the optimal condition. At such optimal particle distribution, those coarse particles are closely packed and the holes in-between those coarse particles are filled by fine ones. In this case, the gap between particles becomes minimized and thus, the lowest linear shrinkage has been achieved during the drying process. In addition, the closely packed particles apparently have some beneficial effects on the mechanical properties.

From Fig.3(d), with the increase of the solid content, the linear shrinkage of the green body is continuously decreasing rather steeply, whilst the flexure strength 
(Fig.4(d)) is continuously increasing slowly. It can be explained that with the increase of the solid content, the gap between particles decreases, and the impact of water loss decreases as a result of less water involved in the system. In addition, after drying procedure, when the water in the gap of particles was gone, particles in the system had already become dense no matter how much the shrinkage was in drying process, so that bending strength rised slightly when solid content increased.

\subsection{Properties of $\mathrm{SiC}$ ceramic by GSI process}

Fig.6 (a) and (b) are the pictures of the green body produced at the optimized gel casting condition and the final product (i.e. the above green body after the GSI process).

Fig.7 shows the load-displacement curve of such final product. As seen, the sample shows a typical brittle fracture behavior. The accordingly calculated flexure strength and elastic modulus are $245 \mathrm{MPa}$ and $220 \mathrm{GPa}$ respectively. Fig. 8 shows the SEM images of fracture surfaces of the final product, confirming that these samples made from such optimized conditions are rather dense, free from cracks, pores or holes.

Fig.9 exhibits the XRD pattern of the made ceramic after the GSI process. As shown, there are three phases present in the final product. They are respectively the cubic silicon, $\beta-\mathrm{SiC}$ and $\alpha-\mathrm{SiC}$ [19]. While the $\beta-\mathrm{SiC}$ is the reaction product of the GSI process, the $\alpha$-SiC originates from the raw materials and the cubic silicon is the unreacted residue silicon during the GSI process.

From XRD spectrum, no obvious peaks referring to carbon were found. The ratio 
of $\alpha-\mathrm{SiC} / \beta-\mathrm{SiC}\left(\mathrm{X}_{\alpha} / \mathrm{X}_{\beta}\right)$ can be calculated according to the $\mathrm{K}$ value method (Eq.6),

$$
\frac{X_{\alpha}}{X_{\beta}}=\frac{I_{\alpha} \cdot K_{\beta}}{I_{\beta} \cdot K_{\alpha}}
$$

where I is diffraction intensity gotten from XRD pattern, $\mathrm{K}$ refers to the $\mathrm{K}$ value of samples where $\mathrm{Al}_{2} \mathrm{O}_{3}$ is used as reference $\left(\mathrm{K}_{\alpha}=1.600, \mathrm{~K}_{\beta}=1.811\right)$, carbon takes 15 wt.\% in solid powders, imaging all the carbon turn to $\beta$-SiC at last, the theoretical value of $\mathrm{X}_{\alpha} / \mathrm{X}_{\beta}$ was 1.73 . From $\mathrm{XRD}$ spectrum, since both the first and second strongest diffraction peaks of $\alpha-\mathrm{SiC}$ and $\beta-\mathrm{SiC}$ coincide in XRD pattern, the third strongest peaks $\left(\alpha-\mathrm{SiC}\right.$ of $34.18^{\circ}$ and $\beta-\mathrm{SiC}$ of $\left.71.78^{\circ}\right)$ are used to calculate $\mathrm{X}_{\alpha} / \mathrm{X}_{\beta}$ value, the conclusion is 1.70 , which is close to theoretic value, suggesting almost all of carbon black takes reaction in the GSI process.

\section{Conclusions}

In the background of the space optical applications, the current study employs a combination of the gel-casting and GSI techniques to produce a dense SiC ceramic. The major conclusions are as follows.

(1) A new initiator AIBA has been found to replace the conventional one in the aqueous gel-casting system that contains carbon black. The optimal conditions for gelation reactions are as follows: the concentration of the initiator is $20 \mu 1$ per gram of slurry, and the temperature and the dwelling time are $70{ }^{\circ} \mathrm{C}$ and 50 minutes respectively. Under this condition, the conversion rate of the monomer AM is $98.77 \%$.

(2) An optimal condition for gel casting has been achieved. That is, the monomer AM content in water at $25 \mathrm{wt} . \%$, the ratio of the monomer AM to the crosslinking agent MBAM at 12 , the ratio of coarse and fine $\mathrm{SiC}$ particles at $3 / 2$, and the solid 
content at $60 \mathrm{vol} . \%$. At this optimized condition, the linear shrinkage of the green body is reduced to $1 \%$ and the flexure strength of the green body reach $59.2 \mathrm{MPa}$.

(3) The flexural strength and elastic modulus of the final $\mathrm{SiC}$ ceramic after the GSI process can reach $245 \mathrm{MPa}$ and $220 \mathrm{GPa}$ respectively. The final product consists of $\mathrm{Si}, \alpha-\mathrm{SiC}$ and $\beta-\mathrm{SiC}$. The results suggest that a $\mathrm{SiC}$ ceramic with very high performance has been successfully fabricated by a combination of the gel casting and GSI techniques.

\section{Acknowledgements}

The authors are grateful to National Science Foundation of China for financial support. The authors are also grateful to Aid Program for Innovative Group of National University of Defense Technology and Aid Program for Science and Technology Innovative Research Team in Higher Educational Institutions of Hunan Province.

\section{References}

[1] H. B, K. B, P. U, et al., Ultra-lightweight C/SiC mirrors and structures, ESA Bulletin 958 (1998) 148-152.

[2] K. M, K. G S., Cesic: engneering material for optical applications, SPIE 5179 (2003) 223-233.

[3] K. M, K. G S, D. M, Short carbon-fiber reinforced ceramic Cesic for optomechanical applications, SPIE 4837 (2003) 576-588.

[4] R. J. Liu, C. R. Zhang, X. Y. Liu, et al., Surface and interface characteristics of SiC coatings 
by chemical vapor deposition, J. Mater. Sci. 40 (8) (2005) 2109-2111.

[5] Q. Li, S. Dong, Z. Wang, et al., Fabrication and properties of 3-D C $\mathrm{f} / \mathrm{ZrC}-\mathrm{SiC}$ composites by the vapor silicon infiltration process, Ceram. Inter. 39 (2013) 4723-4727.

[6] A. Ortona, P. Fino, C. D’Angelo et al., Si-SiC-ZrB 2 ceramics by silicon reactive infiltration, Ceram. Inter. 38 (2012) 3243-3250.

[7] X. Yang, B. Li, C. Zhang, et al., Fabrication and properties of porous slicon nitride wave-transparent ceramics via gel-casting and pressureless sintering, Mater. Sci. Eng. A. 663 (2016) 174-180.

[8] B. Chen, Z. Zhang, J. Zhang, et al., Aqueous gel-casting of hydroxyapatite, Mater. Sci. and Eng. A. 435-436 (2006) 198-203.

[9] K. Liu, C.R. Zhang, B. Li, et al., Synthesis of porous silicon nitride-boron nitride composites by gel-casting and PIP, J. Mater. Eng. Perform. 23 (8) (2014) 2829-2833.

[10] J.L. Yang, J.L. Yu, Y. Huang. Recent developments in gelcasting of ceramics, J. Eur. Ceram. Soc. 31 (14) (2011) 2569-2591.

[11] R. Zhang, R. He, X. Zhang, et al., Microstructure and mechanical properties of $\mathrm{ZrB}_{2}-\mathrm{SiC}$ composites prepared by gelcasting and pressureless sintering, Int. J. Refract. Met. Hard Mater. 43 (2014) 83-88

[12] B. Li, K. Liu, C.R. Zhang, et al., Fabrication and properties of borazine derived boron nitride bonded porous silicon aluminum oxynitride wave-transparent composite . J. Eur. Ceram. Soc., 34 (2014) 3591-3595.

[13] R. He, X. Zhang, P. Hu et al., Aqueous gelcasting of $\mathrm{ZrB}_{2}-\mathrm{SiC}$ ultra high temperature ceramics, Ceram. Int. 38 (2012) 5411-5418. 
[14] Y. Huang, L.G. Ma, J.L. Yang, Improving the homogeneity and reliability of ceramic parts with complex shapes by pressure-assisted gel-casting, Mater. Lett., 30 (2004) 3893-3897.

[15] J. H. Eom, Y. W. Kim, S. Raju, Processing and properties of macroporous silicon carbide ceramics: A review, J. Asia. Cream. Soc. 1 (2013) 220-242.

[16] J. Zhang, D. Jiang, Q. Lin, et al., Properties of silicon carbide ceramics from gelcasting and pressureless sintering, Mater. Des. 65 (2015) 12-16.

[17] Y. Zhang, Z. Yuan, Y. Zhou, Gelcasting of silicon carbide ceramics using phenolic resin and furfuryl alcohol as the gel former, Ceram. Inter. 40 (2014) 7873-7878.

[18] T. Zhang, Z. Zhang, J. Zhang, et al., Preparation of SiC ceramics by aqueous gelcasting and pressureless sintering, Mater. Sci. Eng. A. 443 (2007) 257-261.

[19] Powder diffraction file, Joint Committee on Powder Diffraction Standard.

[20] L. M. Huang, Y. Xiang, F. Cao, et al., The degradation behavior of UHTCs based coatings coated PIP-C/SiC composites in thermal cycling environment, Compo. Part B, 86 (2016) 126-134.

\section{Figures Caption}

Fig.1 Effects of different variables on viscosity of slurry (a) AM content (b) particle size distribution (c) solid content

Fig.2 Effects of AIBA content (a) and temperature (b) on gelling reaction

Fig.3 Effects of different variables on linear shrinkage of green body (a) AM content (b) AM/MBAM ratio (c) particle size distribution (d) solid content

Fig.4 Effects of different variables on bending strength of green body (a) AM content (b) AM/MBAM ratio (c) particle size distribution (d) solid content 
Fig.5 Cross section of green body with different AM contents (a) $15 \mathrm{wt} . \%$ (b) $20 \mathrm{wt} . \%$ (c) $25 \mathrm{wt} . \%$ (d) $30 \mathrm{wt} . \%$

Fig.6 Photograph of $\Phi 60 \mathrm{~mm}$ samples (a) green body (b) ceramic

Fig.7 Load-displacement curve of the $\mathrm{SiC}$ ceramic

Fig. 8 Microstructure of the samples (a) green body (b) ceramic

Fig.9 XRD spectrum of $\mathrm{SiC}$ ceramic 

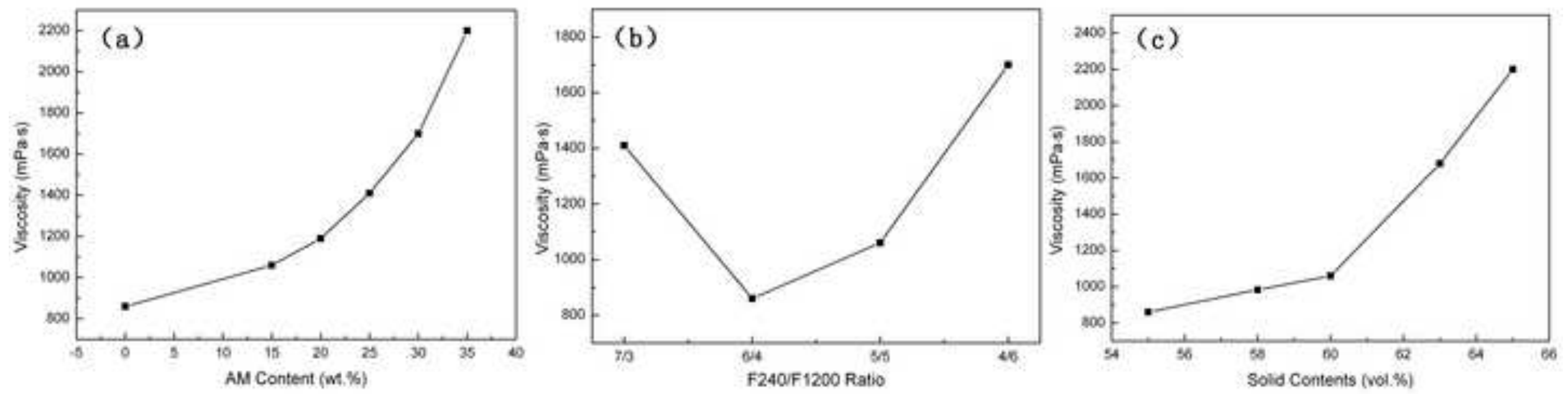

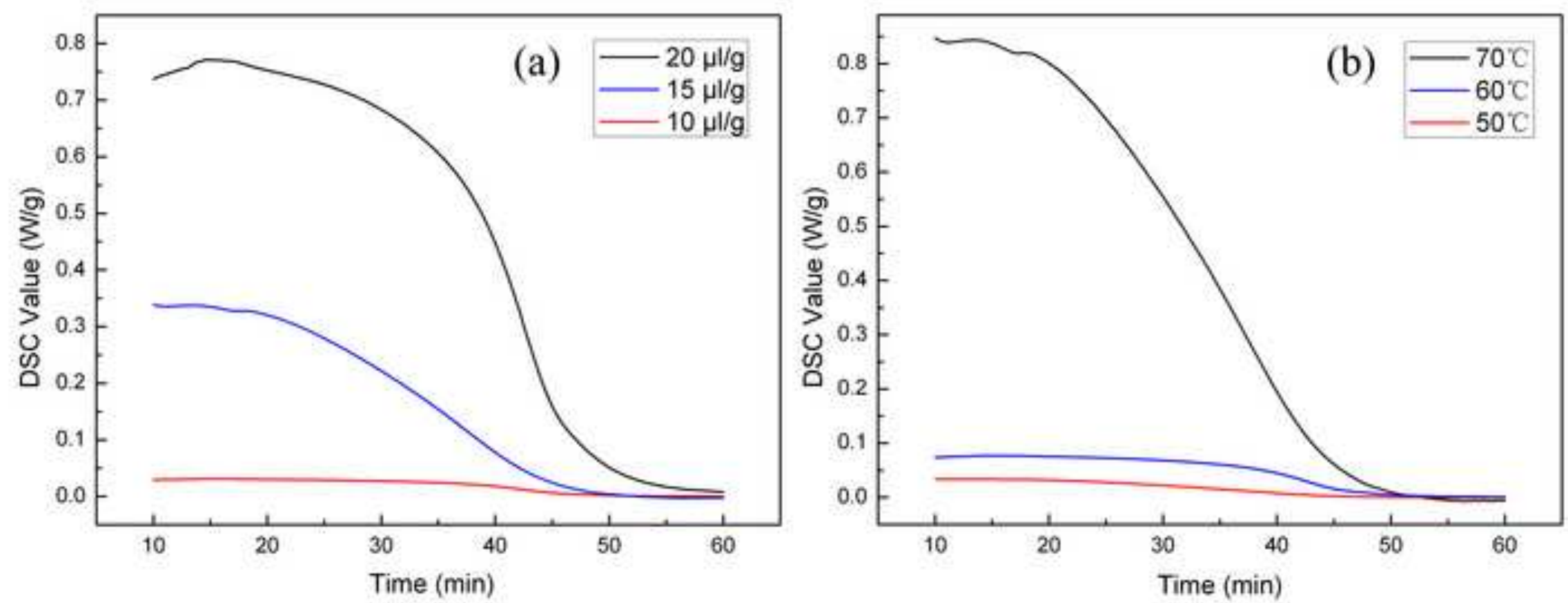

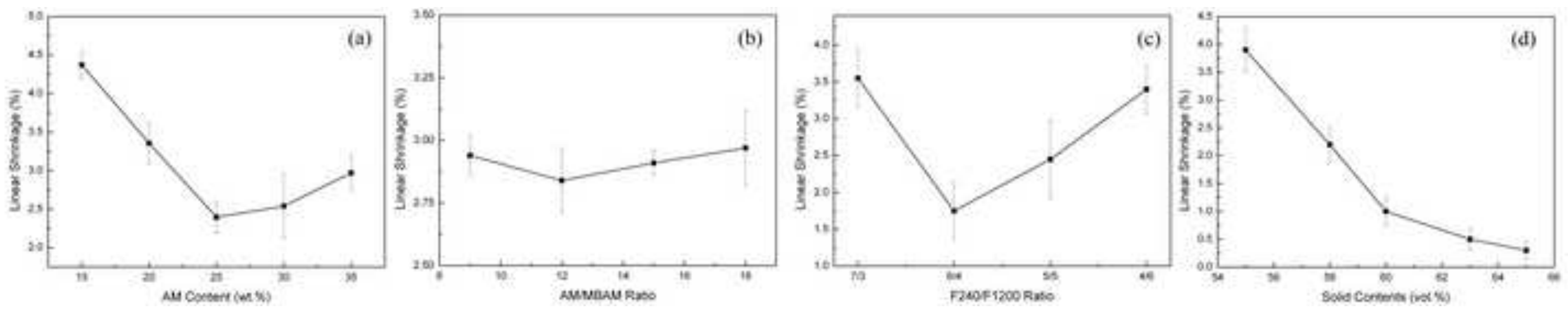

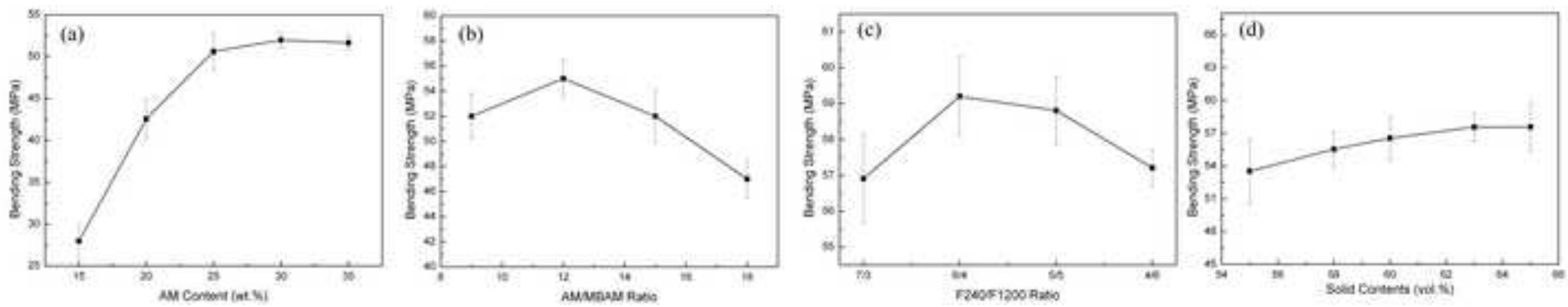


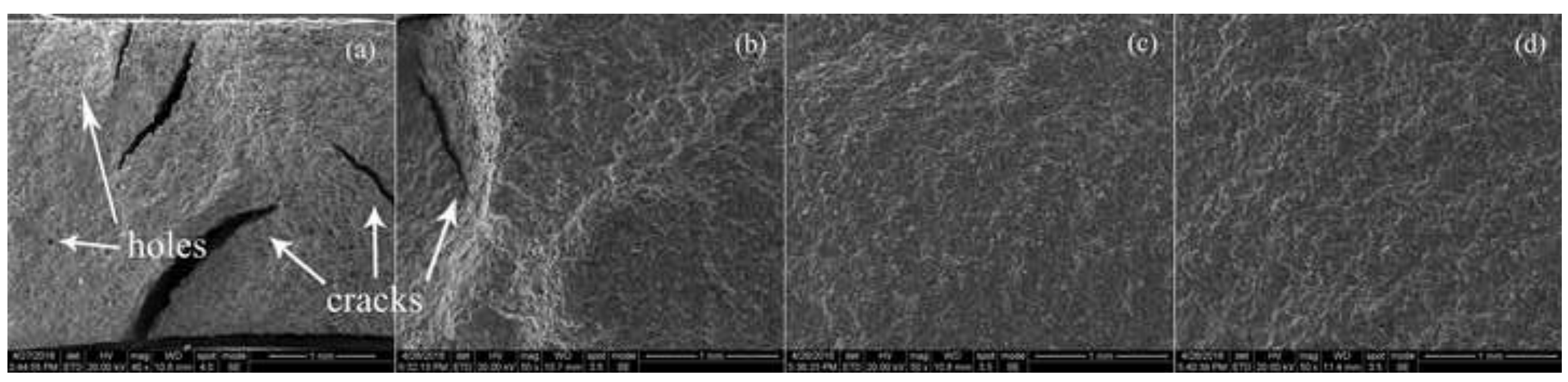




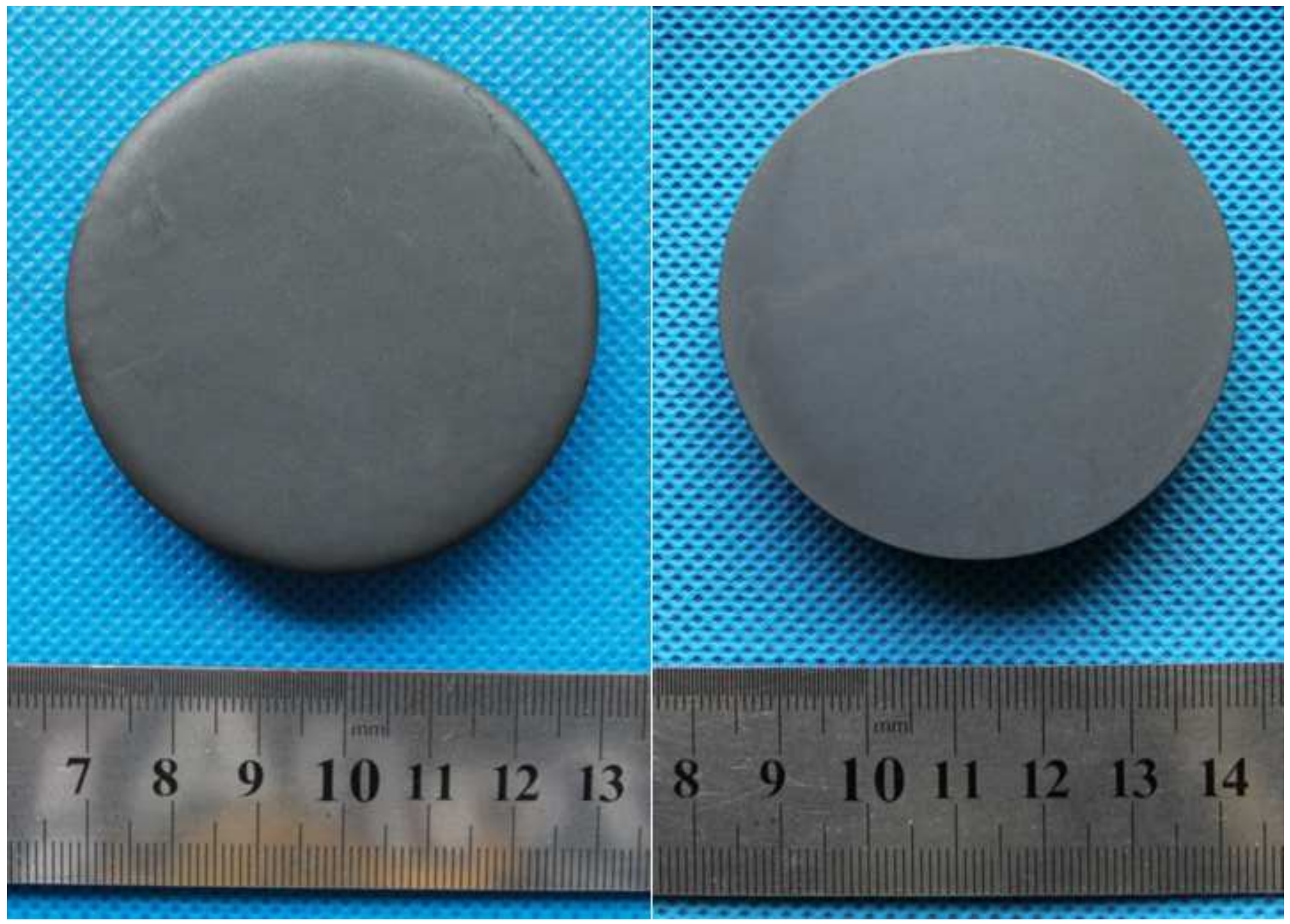




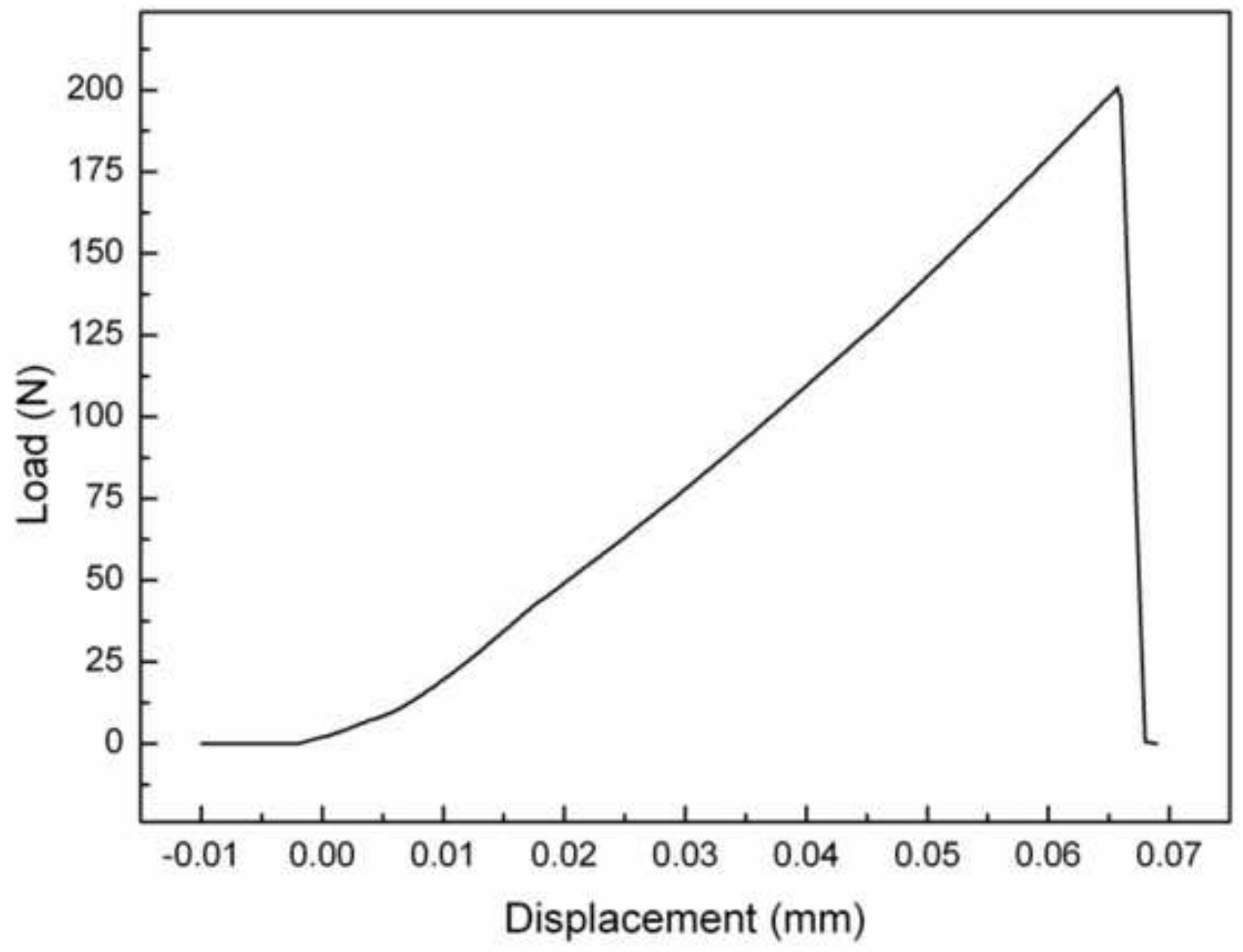




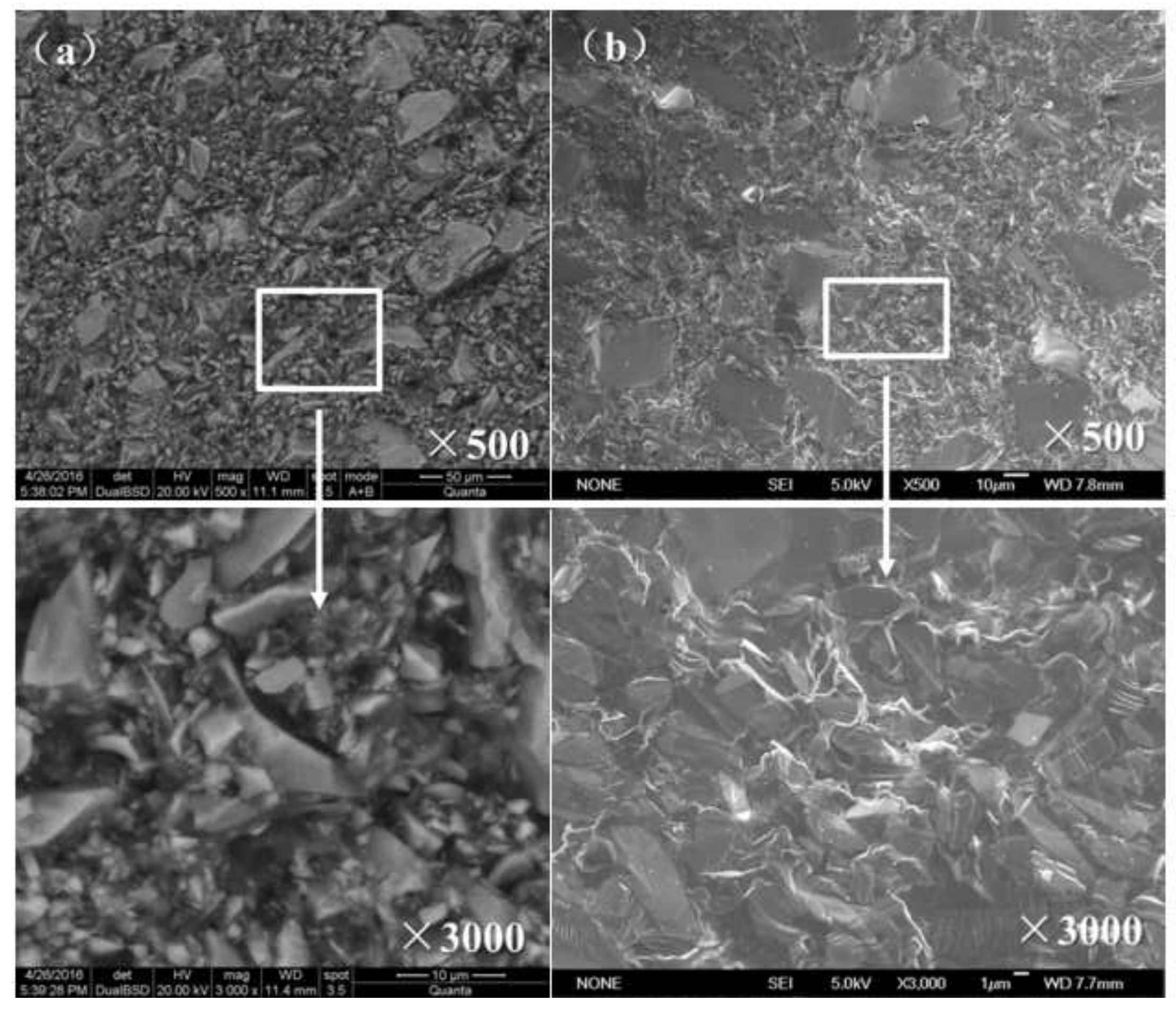




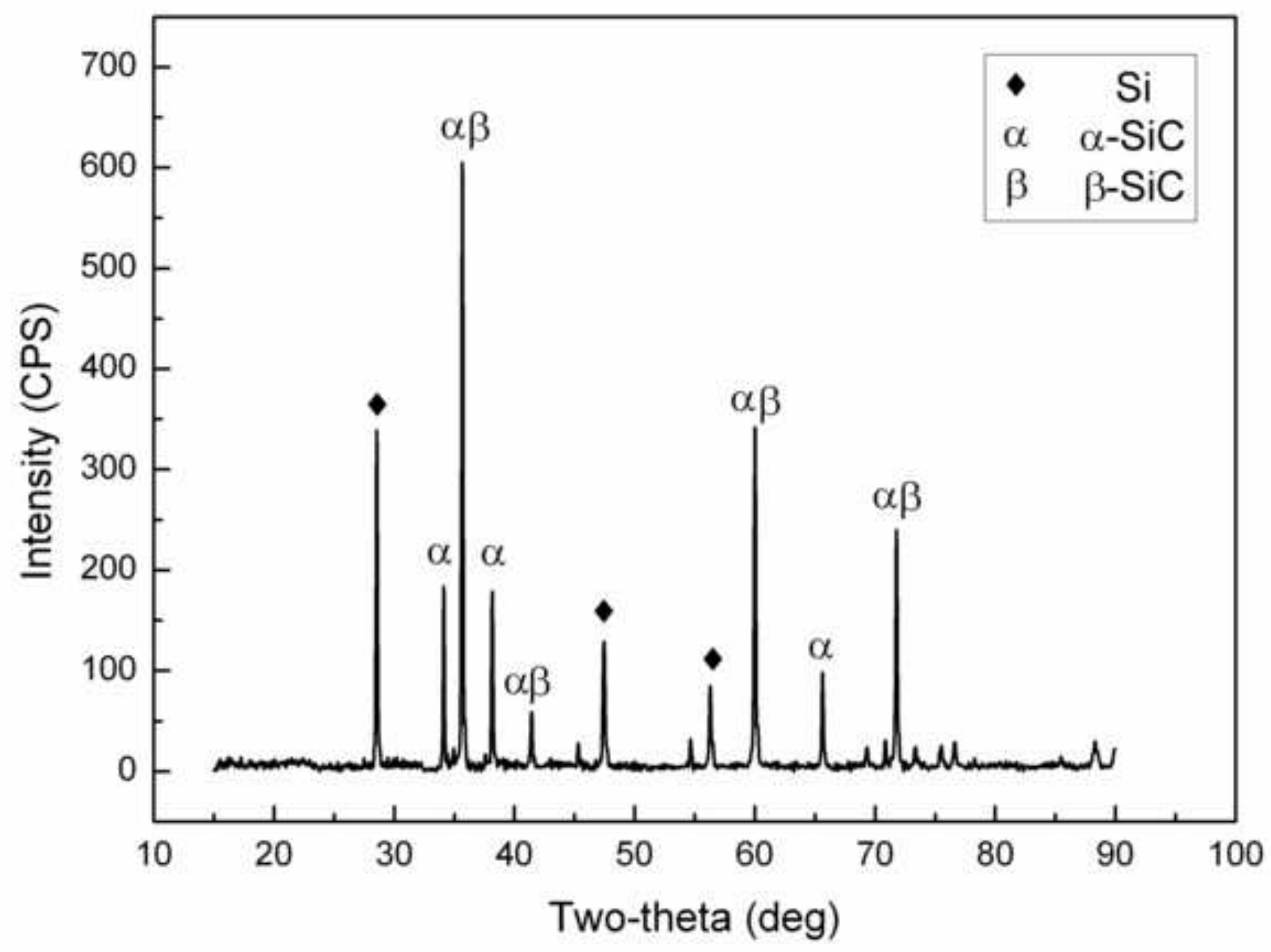

